\title{
Flood risk modeling in the watershed of Charâa stream, Province of Berkane (North-Eastern Morocco)
}

\author{
Hafssa Afif ${ }^{1, *}$, Mostafa Oujidi $^{1}$, Najat Amarjouf $^{2}$, Smahane Ramdani ${ }^{1}$, and Abdellah Azougay ${ }^{1}$ \\ ${ }^{1}$ Applied Geosciences Laboratory "LGA", Department of Geology, Faculty of Sciences Oujda - FSO, Mohammed First University, \\ Oujda. \\ ${ }^{2}$ Research and Development in Engineering Sciences Laboratory "LRDSI", Department of Earth and Environmental Sciences, FSTH, \\ Abdelmalek Essaadi University, Tetouan.
}

\begin{abstract}
The knowledge on floods in Morocco is sporadic and scattered between historical writings and natural events following each other in time and space. In this context, the identification of areas likely to be affected by floods is essential for better management of this risk. The present study aims to model the floods at the level of the two sides of the Charâa stream (the side of Berkane and Sidi Slimane Charâ). The methodology followed in this modeling consists of determining the geomorphology of the watershed and studying its hydrology. 2D hydraulic modeling by Iber software makes it possible to follow the Spatiotemporal evolution of the characteristics of a flood. In addition, it allows us to map the hazard. Furthermore, to assess vulnerability to flooding, we referred to land use (physical vulnerability) to study the behavior of runoff water in the face of socioeconomic issues such as buildings, facilities, and the road network. The results showed that: (1) - A predominance of high-intensity flood hazard in the floodplain; (2) - Land with medium and high vulnerability successively constitutes $46.50 \%$ and $31.10 \%$ of the total studied watershed area.
\end{abstract}

\section{Introduction}

In Morocco, the floods of recent years have been catastrophic in many sites. Several urban areas are currently facing a severe urban management problem due to the flood risks caused by the excessive growth of these urban entities, without adequate sanitation and protection infrastructure[1].

In the Mediterranean region, past floods and forecasts of an increase in their frequency make it necessary for local planners and decision-makers to consider the risk of flooding in territorial management.

In Berkane, urban development in flood zones is one of the main factors aggravating vulnerability. In addition, developments (activities, infrastructure networks) modify flow conditions (waterproofing and runoff) while reducing the flood expansion. On watercourses, the developments (bridge, riprap) and the chronic lack of maintenance on riparian residents aggravate the risk of flooding. Indeed, the occupation of flood areas by buildings and materials sensitive to water can generate, in the event of flooding, the transport and deposition of undesirable products. These products can form obstacles in the stream bed. If they are broken, this can lead to the sudden flooding of downstream areas.

Effective watershed management and the subsequent utilization of water resources have become increasingly important in recent decades, although such management can be complex in rural areas that lack meteorological data. Scientists and engineers around the world have adopted hydrological and hydraulic models for modernday flood forecasting. Hydrological models can estimate flow rates at outlets for various return periods, while hydraulic models can dynamically simulate flow regimes in rivers and flooded valleys.[2]

The present study focuses on the difficulty of flood risk generated by the conjunction of two components of a different nature, hazard and vulnerability. The essential steps in determining this hazard and vulnerability were estimating the maximum flood discharge using regional methods and 2D hydraulic modeling. With this study, we try to present universal methods that could be applied to other similar cities at the local spatial scale. [3]

\section{General Contexts}

\subsection{Geographic Context}

The province of Berkane is located in the extreme NorthEast of the Kingdom and the Oriental region. It is bordered to the north by the Mediterranean Sea, to the east by the Morocco-Algerian border, and the prefecture of Oujda-Angad, to the west by Nador, and the south by the province of Taourirt. Covering a total area of 1985 $\mathrm{Km} 2$, the province of Berkane represents $2.2 \%$ of the region's total area. [3]

* Corresponding author: afif.hafssa@ump.ac.ma 
The diversification of its natural environment, the richness of its cultural heritage, and its privileged geographical location give the province particular assets and potential for agricultural and tourist activities.[4]

\subsection{Geological Context}

The province of Berkane is characterized by contrasting reliefs from North to South: the Triffa Plain, the BéniZnassène mountain range, and the Angad Plain. [4]

\subsection{Hydrographic Context}

A branched hydrographic network characterizes this territory. The Moulouya river is considered the main watercourse of the province and the entire region and other less essential watercourses [5].

This study is based on the hydrological regime of the Charâa Stream. The latter drains three deep and narrow valleys in the Beni-znassène upstream of Berkane: Zegzel gorges, Ouartass and Beni-Ouaklane. However, it is above all the Zegzel Stream that ensures its durability due to sources of Liasic origin. The Cherrâa stream flows on a straight path of $12 \mathrm{~km}$ (the shortest distance between Moulouya and Berkane) to reach Moulouya. Downstream of Berkane, over a distance of about $4 \mathrm{~km}$, it feeds the water table, then beyond this section, it drains the water table to Moulouya.

\subsection{Climatological Context}

The area is characterized by a Mediterranean climate (Köppen classification; Csa); Berkane has an average temperature of $18.2^{\circ} \mathrm{C}$ with a maximum average of $32.4^{\circ} \mathrm{C}$ and a minimum of $6^{\circ} \mathrm{C}$. The total annual rainfall varies between $350 \mathrm{~mm}$ and $530 \mathrm{~mm}$ and is characterized by a Spatio-temporal irregularity. [7].

\section{Materials and Methods}

Risk can be interpreted as the confrontation of hazard and vulnerability in a specific place and at a specific time; hazard being considered as the probability of occurrence of a natural, potentially dangerous, and potentially damaging phenomenon to property and people; and vulnerability as the magnitude of damage or loss resulting from the occurrence of a given natural phenomenon. [9]

This study is carried out according to an adapted scientific protocol using computer tools for data processing:

- The Global Mapper software: a tool for downloading the satellite image of the study area (Berkane region).

- ArcGis 10.6.1 software: GIS tool for digital terrain modeling and land cover digitization.

- The application of the "SCS" method for the quantification of the peak flow.

- The Iber software: a tool for data simulation and 2D modeling of the hazard.

The study is carried out in the following steps:
- DEM preparation and satellite imagery.

- The study of the sub-watershed of Charâa Stream, which includes the cities of Berkane and Sidi Sliman Charâa fed by Ouertass Stream and ZagZel Stream as two main drains by delimiting the sub-watershed, by classifying the rivers on ArcGis by the hydrological modeling tools and by transforming rainfall into flow by the SCS method after deducing its morphological characteristics:

-The surface area of the basin is measured in hectares or square kilometers.

- Length is measured in meters or kilometers.

- The slope is measured as a percentage.

- Concentration time is when it takes for a drop of water to travel the length of the basin. This is the minimum amount of time a rainfall must last for the entire pond to react. It is expressed in hours.

- The production of a land use map that allows an understanding of its organization and spatial dynamics and that is produced from satellite images.

- Drawing up a map of the territory's vulnerability to flooding, which shows the sensitivity of the territory to a hazard and which is based on its physical vulnerability (the resistance of buildings, installations, networks, and infrastructures).

The hazard map development identifies areas where flooding is likely to occur, to a greater or lesser extent and with a greater or lesser frequency. It is based on combining the following two concepts: the velocity of the flood and the depth of water.

\section{Results and Discussion}

\subsection{Hydrology of the sub-basin}

The following figure (Fig.1) shows the delimitation of the sub-watershed of Charâa Stream characterized by:

Table 1. Characteristics of Charâa Stream

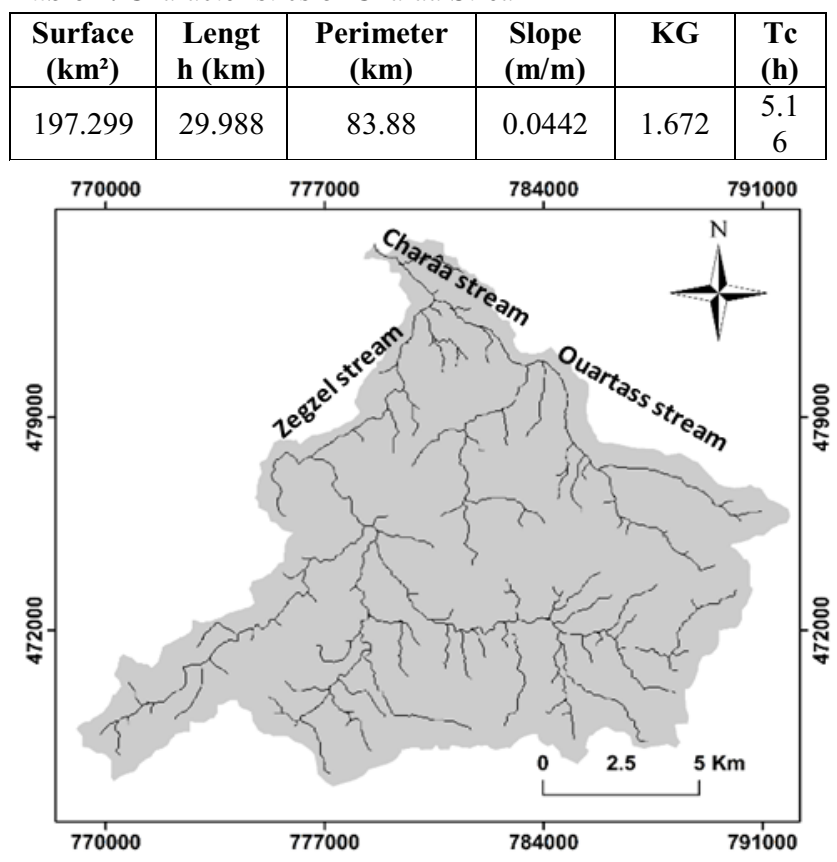

Fig 1. Map of the Charâa Stream sub-watershed 
Applying the SCS method, we obtain the following hydrograph (Fig.2), which presents the instantaneous debits in $\mathrm{m} 3 / \mathrm{s}$, with time in hours with a base time of $14 \mathrm{~h}$, a maximum debit of $580.1 \mathrm{~m} 3 / \mathrm{s}$.

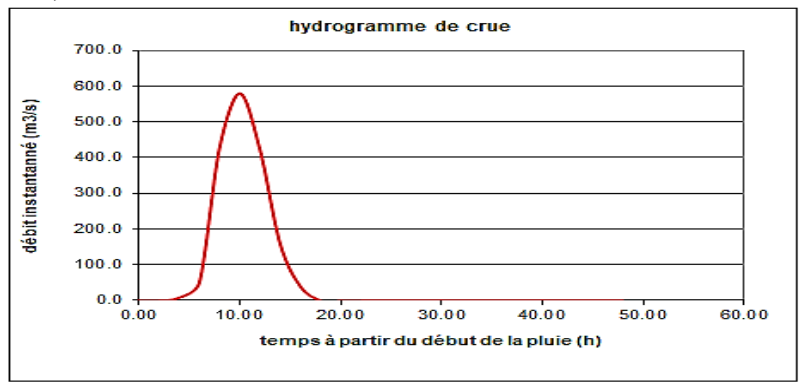

Fig.2. The flood hydrograph (SCS method)

\subsection{Land Use}

The land use map of the study area illustrates the different types of urban, rural, mountainous environments, etc., based on the classification of the Iber 2.5 software (bare soil, dense vegetation, sparse vegetation, industrial zone, infrastructure, residential zone, forest, river, unclassified zone, urban vegetation).

For our problem, this land use (Fig.3) will be used to study the behavior of runoff water as a function of the porosity of each type of soil and to define its vulnerability.

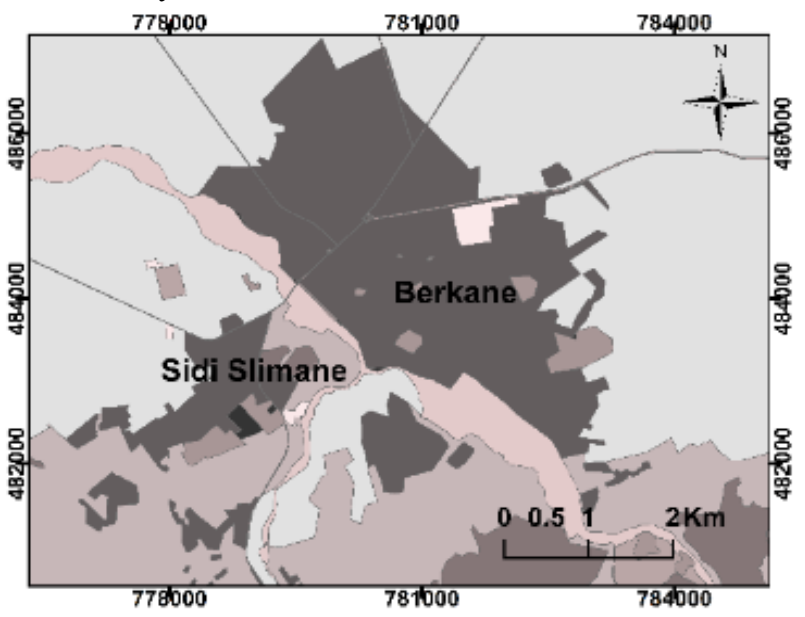

Landuse

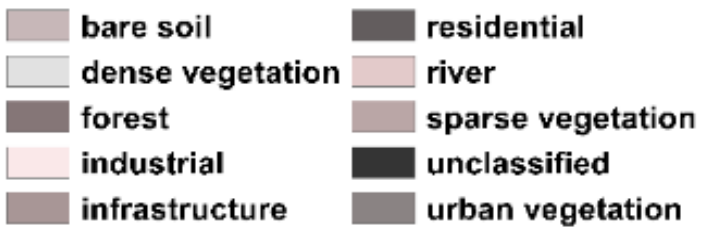

Fig.3. land use map of the study area

\subsection{Vulnerability}

The physical vulnerability for each type of land use takes values between 0 and 1 and between low and high (Table.2), which depends on the importance of this occupation about the presence of residents and goods and infrastructure.
The results obtained by mapping on the ArcGis tool (Fig.7) show that the study area is characterized by a high vulnerability occupying $31.10 \%$ of the studied area and a medium vulnerability occupying $46.50 \%$ of the said area. This high vulnerability can increase the risk of flooding.

Table 2. Vulnerability Indicator

\begin{tabular}{|c|c|c|}
\hline \multirow{2}{*}{ Land use } & \multicolumn{2}{|c|}{ Vulnerability indicator } \\
\cline { 2 - 3 } & from 0 to 1 & in terms \\
\hline $\begin{array}{c}\text { Bare soil/ Sparse } \\
\text { vegetation/ forest/ } \\
\begin{array}{c}\text { Unclassified /Urban } \\
\text { vegetation }\end{array}\end{array}$ & 0 & Low \\
\hline Dense vegetation & 0.5 & Med \\
\hline $\begin{array}{c}\text { Industrial/ infrastructure/ } \\
\text { Residential / rivière }\end{array}$ & 1 & High \\
\hline
\end{tabular}

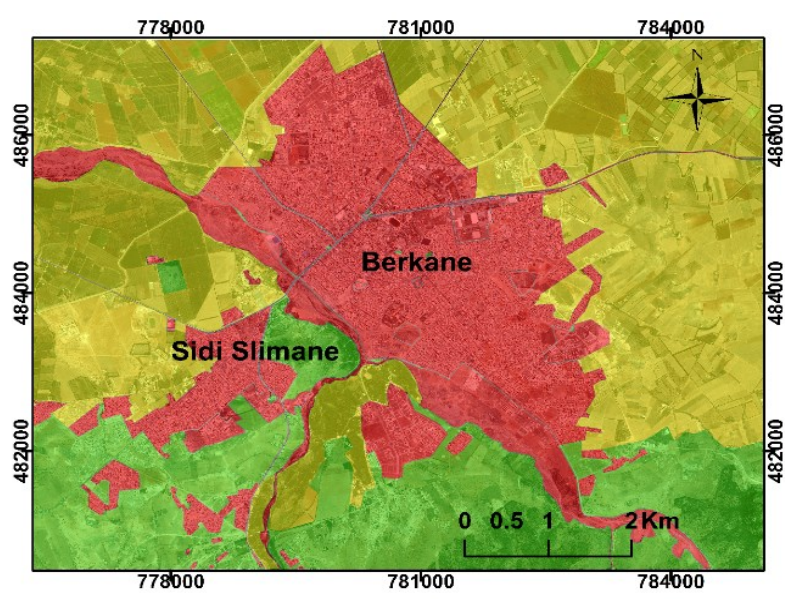

\section{Vulnerability}

\section{Low}

0.5 Medium

1 High

Fig.4. Vulnerability map for Berkane and Sidi Sliman Charâa

\subsection{Hazard}

The hazard map is the result of the hazard study, and the objective is to assess the hazard intensity at any point in the study area.

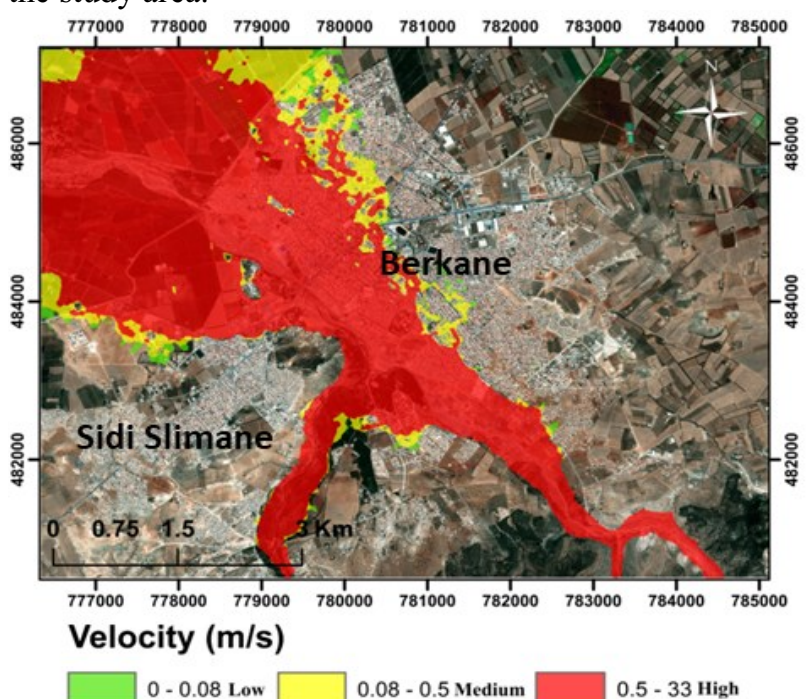

Fig.5. Flow velocity map of Charâa Stream 
The hazard represents flooding as a physical, natural, and uncontrollable phenomenon, regardless of its potential effects on the environment and human activities. Hazard depends on the hydrological behavior of the catchment area and the hydraulic functioning of the river system.

Our first factor in determining hazard is the flow velocity (Fig.5).

Our second factor in determining hazards is water depth (Fig 6).

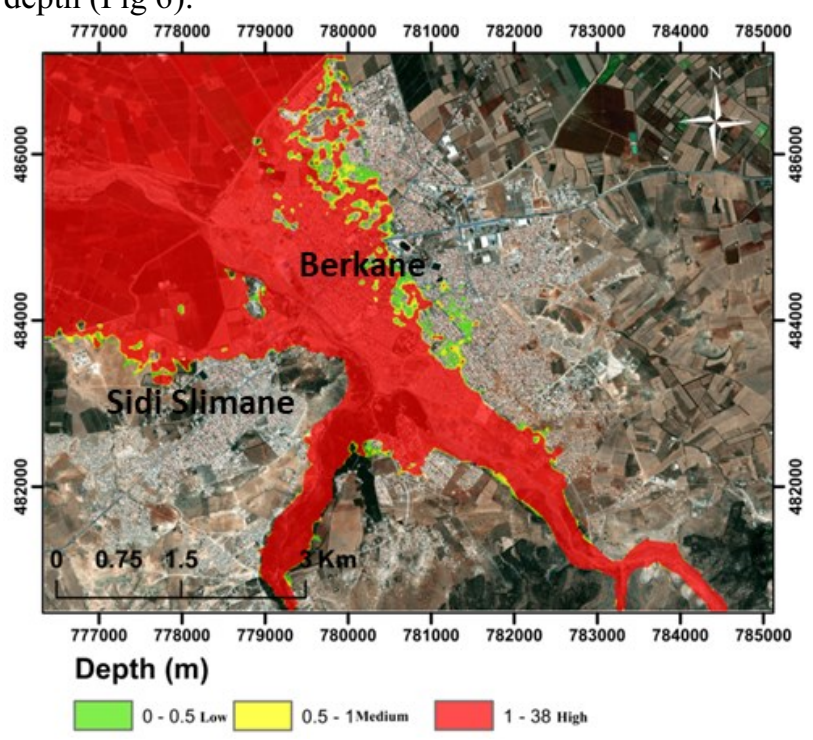

Fig.6. Water depth map of Charâa Stream

After multiplying the two flow velocity maps (Fig. 5) of the flood and the water depth (Fig. 6), we obtain the hazard map using the ArcGIS tool (Fig. 7):

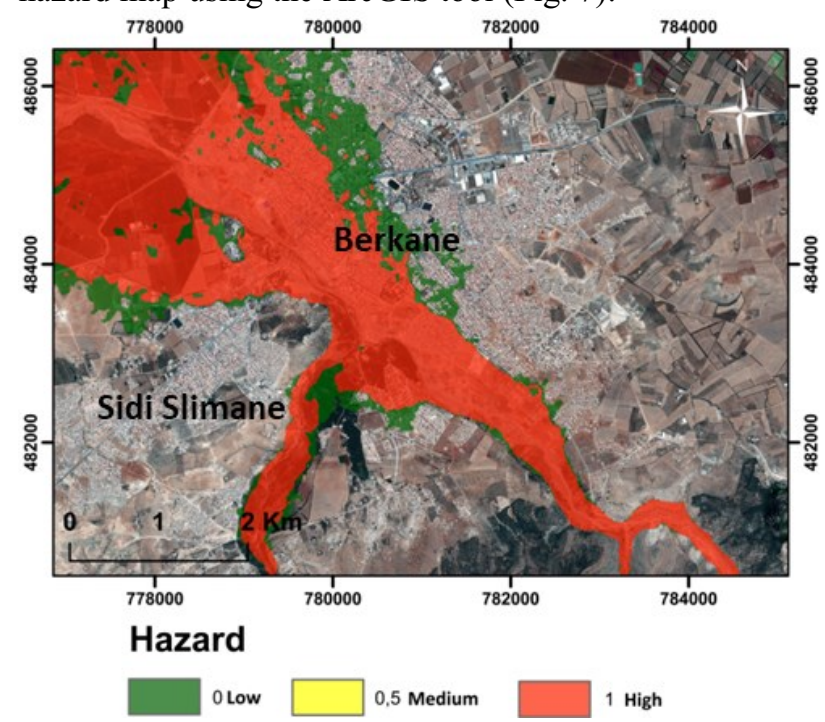

Fig.7. Hazard map for Berkane and Sidi Sliman Charâa

Our study area is characterized by a high flow velocity exceeding $0.5 \mathrm{~m} / \mathrm{s}$ and a significant depth exceeding $1 \mathrm{~m}$. Combining these two factors gives an extreme hazard over a large area at the level of the Berkane city and Sidi Slimane Charâa. This result gives us an idea of the danger under which the region is exposed in the case of a centennial flood.

\section{Conclusions}

The study of hazard and vulnerability on the site of Berkane and Sidi Sliman Charâa highlights the physical characteristics of the floods and their stakes. Urban development aggravates the flooding phenomenon of Stream Charâa, which exposes a significant population facing this risk and the infrastructures of the area.

The knowledge of hydrological mechanisms and human occupation of the land is insufficient to solve flooding in Berkane and Sidi Slimane Charâa. On the other hand, it is only a first step towards better risk management, particularly by proposing competent authorities cartographic tools for decision support.

\section{Acknowledgments}

This study was carried out thanks to the excellence grant from the National Center for Scientific and Technical Research (CNRST)

\section{References}

1. D. Elhafid. B. Akdim. Echelle Spatiale Et Genese Des Risques Hydrologiques En Milieu Urbain Et Peri-Urbain : Cas De La Ville D ’ Oujda. 10 (2004).

2. N. Amarjouf. M. Oujidi. D. Meijer. W. Klop. ISH Hydraul Eng. Evaluating ephemeral watercourse discharges by hydrologic-hydraulic modeling: application to the Kert River basin, Morocco. 11. (2019).

3. Z. Naiji. M. Oujidi. N. Amarjouf. and H. Rezqi. Application of Two-Dimensional Hydraulic Modeling in Flood Risk Mapping. A Case of the Urban Area of Zaio, Morocco. 22. (2019)

4. Ministère de l'Aménagement du Territoire National, de l'Urbanisme, de l'Habitat et de la Politique de la Ville. Appel d'Offres Ouvert N ${ }^{\circ} 25$ / 2018 ETUDES RELATIVES A L'ELABORATION DES CARTES D'APTITUDE A L'URBANISATION LOT $\mathrm{N} \circ 1$ : RELATIF A LA PROVINCE DE BERKANE. 33. (2018).

5. MINISTETRE DE L'INTERIEUR/ PROVINCE DE BERKANE. La province de Berkane en chiffres 1. 33. (2006).

6. S. MILHI. A. SBAI. K. BENRBIA. Les enjeux sociaux du tourisme dans la province de Berkane. 21. 113-134. (2018)

7. N. Bechler-Carmaux. M. Mietton. M. Lamotte. Ann. Georgr . Le risque d'inondation fluviale à Niamey (Niger). Aléa, vulnérabilité et cartographie. 11. 176187. (2020). 